SUNY-NTG-03/03

\title{
DEVELOPMENTS IN RANDOM MATRIX THEORY
}

\author{
P.J. Forrester ${ }^{1}$, N.C. Snaith ${ }^{2}$ and J.J.M. Verbaarschot ${ }^{3}$
}

\author{
${ }^{1}$ Department of Mathematics and Statistics, \\ University of Melbourne, Victoria 3010, Australia \\ email: P.Forrester@ms.unimelb.edu.au \\ ${ }^{2}$ School of Mathematics, University of Bristol, \\ University Walk, Clifton, Bristol BS8 1TW, UK \\ email: N.C.Snaith@bristol.ac.uk \\ ${ }^{3}$ Department of Physics and Astronomy, SUNY Stony Brook, \\ Stony Brook, NY 11790, USA \\ e-mail: jacobus.verbaarschot@stonybrook.edu
}

\begin{abstract}
In this preface to the Journal of Physics A, Special Edition on Random Matrix Theory, we give a review of the main historical developments of random matrix theory. A short summary of the papers that appear in this special edition is also given.
\end{abstract}




\section{Introduction}

Random matrix theory has matured into a field with applications in many branches of physics and mathematics. A large number of physicists and mathematicians have been fascinated by this subject that has turned out to be surprisingly rich and far reaching. Paraphrasing Dyson, random matrix theory is a new kind of statistical mechanics where the realization of the system is not relevant. Instead of having an ensemble of states we have an ensemble of Hamiltonians. Ergodicity is now the equivalence of spectral averaging and the averaging over this ensemble.

Random matrix theory has been particularly successful in three areas: first, in describing level correlations on the scale of the average level spacing; second, in providing the generating function for combinatorial factors of planar diagrams; and, third, as an exactly solvable model with intricate connections to the theory of integrable systems. One of the reasons for the success of random matrix theory is universality: eigenvalue correlations on the scale of the average level spacing do not depend on the probability distribution. This property is at the very foundation of random matrix theory. It suggests that random matrix theory correlations of eigenvalues should be the rule rather than the exception. However, the most important reason for studying random matrix theory is that its predictions do occur in nature in systems as varied as nuclear energy levels, zeros of the Riemann $\zeta$ function and sound waves in quartz crystals. Another of the roles of random matrix theory is that the large $N$ limit of its partition function is a generating function for planar diagrams which have played an important role in quantum field theory. For example, they are the leading contributions to QCD with a large number of colors, and they are dual to triangulations of a random surface and thus describe two-dimensional quantum gravity. In addition to this, random matrix theory has attracted a great deal of interest because of the mathematical challenges it poses. The problems are highly nontrivial, but, with sufficient effort, many of the questions that arise in this field can be answered in full.

It is hard not to be fascinated by random matrix theory. Everyone who works in this field has experienced the amazement of obtaining truly universal behavior by diagonalizing a large random matrix. Nowadays, as we can see from the contributions to this special edition, the excitement about the subject is still as much alive as when it was first created. In the introduction of this special edition we give a short summary of the history of random matrix theory. The historical perspective is certainly colored by our personal experience, and, for a somewhat different perspective, we refer to the introduction in the review by the Heidelberg group [1]. We conclude with a short overview of the papers that will appear in this special issue. 


\section{History of Random Matrix Theory}

Random matrix theories have fascinated both mathematicians and physicists since they were first introduced in mathematical statistics by Wishart in 1928 [2]. After a slow start, the subject gained prominence when Wigner [3] introduced the concept of statistical distribution of nuclear energy levels in 1950. However, it took until 1955 before Wigner [4] introduced ensembles of random matrices. In this paper he also introduced the large $N$ expansion and realized that the leading order contribution to the expectation values of moments of the random Hamiltonian is given by planar diagrams. In 1956, Wigner [5] derived the Wigner surmise from the level spacing distribution of an ensemble of $2 \times 2$ matrices after level repulsion had been predicted by Landau and Smorodinksy [6] and observed by Gurevich and Pevsner [7]. The idea of invariant random matrix ensembles was introduced in physics by Porter and Rosenzweig [8] after it had appeared earlier in the mathematical literature. A mathematically rigorous analysis of spacing distributions was first given by Gaudin [9] and Mehta [10]. To analyze the eigenvalue density Mehta [11] invented the orthogonal polynomial method.

The mathematical foundations of random matrix theory were established in a series of beautiful papers by Dyson [12]-[16]. He introduced the classification of random matrix ensembles according to their invariance properties under time reversal $[12,16]$. As we all know, only three different possibilities exist: a system is not time reversal invariant, or a system is time reversal invariant with the square of the time reversal invariance operator either equal to 1 or -1 . The matrix elements of the corresponding random matrix ensembles are complex, real and self-dual quaternion, respectively, which from a mathematical viewpoint exhaust the distinct real commutative normed division algebras, or in effect number systems. The corresponding invariant Gaussian ensembles of Hermitian random matrices are known as the Gaussian unitary ensemble (GUE), the Gaussian orthogonal ensemble (GOE) and the Gaussian symplectic ensemble (GSE), in this order.

Dyson [12] also formulated the underlying philosophy of random matrix theory. In his words, "What is here required is a new kind of statistical mechanics, in which we renounce exact knowledge not of the state of the system but of the system itself. We picture a complex nucleus as a "black box" in which a large number of particles are interacting according to unknown laws. The problem then is to define in a mathematically precise way an ensemble of systems in which all possible laws of interaction are equally possible". This was made more precise by Balian [17], who obtained the Gaussian random matrix ensembles from minimizing the information entropy.

A second important result of Dyson's papers $[14,15]$ was the relation between random matrix theory and the theory of exactly integrable systems: the partition function of a random matrix ensemble is equivalent to the partition function of a log-potential Coulomb gas in one dimension at three special tem- 
peratures, each with solvability properties not shared for general temperatures. Moreover the evolution of the eigenvalues of parameter dependent extensions of the Gaussian ensembles was shown to be controlled by a Fokker-Planck operator which also specifies the Brownian evolution of the Coulomb gas. These results were further explored by Sutherland [18] when he realized that the CalogeroSutherland quantum many body system $[19,18]$, for which the Hamiltonian can be constructed from $N$ independent commuting operators and so is integrable, is mathematically equivalent to Dyson's Brownian motion model. The relation between random matrix theory and integrable systems is discussed extensively in the monograph by Forrester [20]. A review of one dimensional integrable systems that touches on many ideas that also appear in random matrix theory is given in the book by Korepin, Bogoliubov and Izergin [21]. A third idea that appeared in Dyson's paper [12] is the application of Shannon's information entropy to random matrix spectra.

The early developments in random matrix theory are well summarized in the first edition of the monograph by Mehta [22]. This has been a very influential book containing many mathematical details which have been proved to be extremely useful over the years. A second significant book is by Porter [23]. It contains reprints of the important papers on random matrix theory that were written before 1965 .

About the same time as the early development of random matrix theory in nuclear physics, the field of disordered systems was born by the work by Anderson [24] on the localization of wave functions in one dimensional disordered systems. He considered a one dimensional lattice with a random potential at each lattice point. He found that the eigenfunctions of this system are exponentially localized. His work had strong impact on both experimental and theoretical solid state physics. Another early application of random matrix theory is the theory of small metallic particles by Gorkov and Eliasberg [25], which nowadays would be part of mesoscopic physics.

Random matrix theory, which was first formulated in mathematical statistics, continued to develop in mathematics independently of the developments in physics. Important results with regards to the integration measure of invariant random matrix ensembles were obtained by L.K. Hua [26]. His results of more than a decade of work are summarized in his book that appeared in 1959 but which remained largely unknown. Only a small number of mathematicians worked on integrals that appear in random matrix theory. One very important result was obtained by Harish-Chandra [27], who evaluated a unitary matrix integral that is now known as the Harish-Chandra-Itzykson-Zuber integral [27, 28]. Zinn-Justin and Zuber [140] review this topic in the present special issue. Also the work of Selberg [29] is well-known, not in the least because Madan Lal Mehta devoted a chapter of the second edition of his book [30] to this subject. Another noteworthy contribution is the introduction of zonal polynomials by James [31]. The 1982 book of Muirhead [32] ties together matrix integrals and zonal polynomials as they are relevant in mathematical 
statistics. Girko has written a number of mathematical books (see e.g. [33]) relating to analytic properties of the eigenvalue distribution of large random matrices. Voiculescu [34] used random matrices as a primary example of the concept of free non-commutative random variables in operator algebras. However, the mathematical literature remained largely unnoticed by physicists until recently.

What is more surprising is that the theory of disordered systems and the application of random matrix theory in nuclear physics proceeded more or less independently until the seminal work by Efetov on the supersymmetric method [35] and its application [36] to the theory of small metallic particles and to localization theory [37] . This is even more remarkable since both the papers by Anderson and Dyson were written at Princeton.

The main developments in random matrix theory in the decade after the appearance of the first edition of Mehta's book were applications to nuclear physics. In particular, the statistical theory of $S$-matrix fluctuations received a great deal of attention. The first work in this direction dates back to Wigner [38], who simultaneously studied the distribution of the widths and the spacings of nuclear resonances, and to Porter and Thomas [39], who introduced the Porter-Thomas distribution for nuclear decay widths. Correlations of crosssections at two different energies were considered in [40] and are now known as Ericson fluctuations. The formulation of the theory of $S$-matrix fluctuations was completed in the work of Agassi et al. [41] . In this paper the authors introduced resummation techniques which later received much more attention in the field of impurity scattering, introduced earlier in the book by Abrikosov, Gorkov and Dzyaloshinski [42]. The problem of the distribution of poles of $S$-matrices was also the motivation of Ginibre [43] for introducing what is now known as the Ginibre ensemble with eigenvalues uniformly distributed inside a disk in the complex plane. His paper initiated the subfield of nonhermitian random matrix theory which is reviewed by Fyodorov and Sommers [148] in this issue.

In 1973 Montgomery [44] made a conjecture for the asymptotic limit of the two-point correlation function of the zeros of the Riemann $\zeta$ function on the critical line. Together with Dyson he realized that his conjectured result is the two-point function of the GUE. The connection was extended to higher correlation functions of the Riemann zeros by Hejhal [45] and Rudnick and Sarnak [46], although the full correspondence of the correlation functions with random matrix theory has still not been proved. A heuristic derivation of these results using the Hardy-Littlewood conjecture for the correlation between primes, was given by Bogomolny and Keating in 1995 [47, 48]. Mathematically rigorous results relating the two-point functions for the zeros of families of finite field zeta functions and eigenvalues of random matrices from the classical groups are the topic of the monograph by Katz and Sarnak [49].

The conjectured correspondence of the statistics of these zeros of the Riemann $\zeta$ function with the $n$-point correlation function of random matrix eigenvalues has recently meant that random matrix theory has become very useful 
for conjecturing quantities in number theory that were previously unattainable by any method. These include mean values of the Riemann zeta function and other $L$-functions [50]-[55], the order of vanishing at special values of $L$-functions [52], as well as discrete moments of the derivative of the Riemann zeta function $[53,125]$ and the horizontal distribution of the zeros of the derivative [127]. For more details there is a review in this issue by Keating and Snaith [128].

In the period 1975-1985, random matrix theory developed rapidly and became unified with the theory of disordered systems. The first step in this direction was made by Edwards and Anderson [56] who, in their influential paper on spin glasses, introduced the replica trick. This provided a natural framework for a field theoretical formulation of the Anderson model which was introduced a few years later by Wegner in 1979 [57]. In this formulation, symmetries and the spontaneous breaking of symmetries led to a new paradigm in the theory of Anderson localization $[58,59,60]$. It was soon realized that the replica formulation only works well for perturbative calculations. This problem was solved by the introduction of the supersymmetric method [35]. In this method the determinants in the generating function of the resolvent are quenched by taking a ratio of two determinants instead of the $n \rightarrow 0$ limit of the $n$ 'th power of the determinant. Relying on earlier work by Wegner [57] using the replica trick, Efetov showed that the partition function of a disordered system is given by a supersymmetric nonlinear $\sigma$-model. He identified a domain of energy differences where the kinetic term of the non-linear $\sigma$ model can be neglected. In this domain the two-point correlation functions coincide with the results derived by Dyson. The energy scale below which the partition function is dominated by zero momentum modes is known as the Thouless energy [61].

The supersymmetric method has been very fruitful. Efetov [35] obtained new results for one-dimensional disordered wires. Exact results were obtained for the theory of $S$-matrix fluctuations [62]. Relations between the orthogonal and symplectic symmetry classes were derived [63] from the supersymmetric partition function. In the subsequent years many more new results were derived by means of the supersymmetric method. Among others we mention results for parametric correlations [64] where eigenvalue correlations for different values of an external parameter are considered. An elaborate discussion of applications of the supersymmetric method to disordered systems is given in the book by Efetov [65].

Exact results for $S$-matrix fluctuations were obtained in a completely independent way by a Mexican group [66]. The exact distribution function of $S$-matrices was found starting from the three assumptions of analyticity, ergodicity and maximizing the information entropy [17]. Another effort in nuclear physics was the introduction of random matrix ensembles that reflected the few-body nature of the interaction. In particular, French and co-workers have pursued this direction of research (see [67] for a review). In this issue Benet and Weidenmueller [162] review recent progress in this field.

A major development was the experimental discovery of universal conduc- 
tance fluctuations by Webb and Washburn in 1986 [68] after having been predicted theoretically by Altshuler [69] and Stone and Lee [70, 71]. This discovery started the new field of chaotic quantum dots. The transport properties of these quantum dots could be described by the supersymmetric nonlinear $\sigma$-model that had been used for the theory of $S$-matrix fluctuations in compound nuclei. In fact, a compound nucleus is a chaotic quantum dot (see [72, 73] and [143] in this issue for reviews).

A few years before the discovery of universal conductance fluctuations, random matrix theory was applied to quantum field theory. Through the work of 't Hooft [74] we know that in the limit of a large number of colors, the QCD partition function is dominated by planar diagrams. This is also the case for the large $N$ limit of random matrix theory. In [75] this was exploited to calculate the combinatorial factors that enter in the large $N_{c}$ limit of QCD by means of random matrix theory. A second innovative idea that appeared in this paper is the formulation of the calculation of the resolvent in random matrix theories as a Riemann-Hilbert problem. This approach has received more attention in the recent mathematical literature [76].

Random matrix theory has had impact on several areas of quantum field theory: lattice QCD, two dimensional gravity, the Euclidean Dirac spectrum and the Seiberg-Witten [77] solution of two dimensional supersymmetric gauge theories. An important result is the Eguchi-Kawai [78] reduction. These authors show that in the limit of a large number of colors, certain gluonic correlation functions of pure Yang-Mills theory can be reduced to an integral over 4 unitary matrices. In two spatial dimensions this reduction results in an integral over a single unitary matrix which can be evaluated in the large- $N$ limit.

A unitary matrix integral also occurs in the low-energy limit of QCD. Because of the spontaneous breaking of chiral symmetry, its low-energy degrees of freedom are the Goldstone modes which are parameterized by a unitary matrix valued field [79]. Below the Thouless energy for this system the kinetic term of the effective Lagrangian can be neglected and the low-energy limit of the QCD partition function is given by the unitary matrix integral [80]. In this domain the eigenvalues of the Dirac operator are correlated according to a random matrix theory with the additional involutive (chiral) symmetry of the QCD Dirac operator $[81,82]$. The same symmetry is also found in two-sublattice disordered systems where hopping only occurs in between the sublattices [83]. The eigenvalue spectrum around zero of these chiral ensembles was first derived in [84]. An important difference between two-sublattice systems and QCD is the topology of the random matrix (i.e. the number of exact zeros) and the fermion determinant. In two-sublattice systems one is only interested in quenched results at zero topology whereas in QCD the fermion determinant and its zero modes are essential. Also in the case of the chiral ensembles we have three different symmetry classes depending on the reality content of the matrix elements. Most of the work on chiral random matrix theory and its applications to the Dirac spectrum in QCD was done in the second half of the nineties (see 
[85] for a review).

In the theory of disordered superconductors four more random matrix ensembles can be introduced [86, 87], thus distinguishing a total of 10 random matrix ensembles. It was noticed by Dyson [88] that each of the three WignerDyson random matrix theories corresponds to a symmetric space. Zirnbauer [89] showed that this observation can be generalized to all 10 symmetry classes of random matrix ensembles with a one-to-one correspondence to each of the large families in the Cartan classification of symmetric spaces.

There have been other attempts to derive QCD from a matrix model. Perhaps best known is the induced QCD partition function of Kazakov and Migdal [90] where the lattice gauge field is coupled to an adjoint scalar field. The gauge field can be integrated out by means of the Harish-Chandra-Itzykson-Zuber integral resulting in a partition function for the eigenvalues of the adjoint scalar field. This partition function can be evaluated by saddle point methods in the large $N$ limit. More recently, it has been shown that that the so-called prepotential of $N=2$ supersymmetric theory can be derived from the large $N$ limit of a random matrix theory [91].

The partition function of $2 \mathrm{~d}$ gravity is a sum over random surfaces which can be described by means of a triangulation [92, 93]. The sum over triangulated surfaces can be written in terms of a random matrix theory partition function. It has been conjectured $[94,95,96]$ that the double scaling limit of this theory describes the continuum limit of the $2 \mathrm{~d}$ gravity partition function. This field brought two new ideas into random matrix theory: universality [97, 98, 99], i.e. that observables are independent of the probability potential, and the connection with integrable systems. In the context of quantum gravity it is natural to consider an arbitrary polynomial probability potential. Integrable hierarchies were obtained from differential equations in the coefficients of the probability potential $[100,101]$. A good review of this topic was given by Di Francesco, Ginsparg and Zinn-Justin [102].

Earlier integrable hierarchies entered in a completely different way. In 1980 it was found by the Kyoto school [103] that the probability of a gap free interval in the infinite GUE is a $\tau$-function for a completely integrable system specifying the isomonodromy deformation of a coupled system of linear differential equations. This had the consequence that the spacing distribution could be expressed in terms of a Painlevé $\mathrm{V}$ transcendent. Later it was found that the distribution of the largest eigenvalue in the GUE is given by the solution of a Painlevé II equation [104]. This development found application in the solution of a long standing mathematical problem: specifying the limiting distribution of the longest increasing subsequence length of a random permutation [105]. In fact the sought distribution is the same as that for the largest eigenvalue in the GUE. The increasing subsequence problem can equivalently be formulated as the polynuclear growth model in $1+1$ dimension [106], and similar relationships with random matrix fluctuations are also known for certain tiling problems [107]. 
The question of why random matrix theory works has been addressed from many different points of view. It was realized early on that the detailed properties of eigenvalue correlations do not depend on the specifics of the probability distribution. One important reason for random matrix theory to work is already mentioned in a work of Dyson [12], asserting that if a system is sufficiently complex, the state of the system is no longer important. However, it took until the early eighties before it was realized that the key reason is that the corresponding classical system is chaotic. Although there have been a few earlier studies relating random matrix theory correlations to classical chaos [108, 109], it was formulated explicitly in a ground breaking paper by Bohigas, Gianonni and Schmit [110] who, based on a numerical study of the Sinai billiard [111], conjectured that level correlations on the scale of the average level spacing are given by random matrix theory if the corresponding classical system is chaotic. This conjecture has been confirmed for numerous systems. The reverse was also shown numerically to be true: if the system is not completely chaotic, the spectral correlations are not given by the Wigner-Dyson ensembles $[112,113]$. Although a complete proof of this conjecture is still lacking, a considerable amount of analytical understanding has been obtained on the basis of a semiclassical analysis $[114,115]$. These inter-relations mean that random matrix theory plays an essential role in the study of quantum chaos, a fact which is given prominence in the books by Haake [116] and Stoeckmann [117].

In this short historical overview we have seen that random matrix theory has been applied to wide ranging fields. Its scope has by far not yet been exhausted as illustrated by recent publications that are as varied as applications to financial correlations $[118,119]$ and wireless communication [120].

\section{The Special Issue}

The present special edition represents a broad cross section of the current activity in random matrix theory. We have subdivided the forty-four contributions into eight different groups: Applications to Number Theory, Applications to Statistical Mechanics, Integrable Systems and Random Matrix Theory, Integration Formulas, Mesoscopic Physics and Disordered Systems, Non-Hermitian Random Matrix Theories, Quantum Chaos, and Special Random Matrix Ensembles.

The contributions to the section "Applications to Number Theory" address the connection between random matrix theory and the Riemann zeta function and other $L$-functions. In [121] and [122] (this second in conjunction with companion papers $[123,124]$, published elsewhere) this connection is examined through the comparison of statistics of the $L$-function zeros with random matrix eigenvalues, while [125], [126] and [127] make use of a comparison between the characteristic polynomial of a random matrix and the $L$-functions themselves. The present status of the application of random matrix theory to $L$-functions is reviewed by Keating and Snaith [128]. 
In section two we discuss applications of random matrix theory to statistical mechanics. Three of the papers $[129,130,131]$ can be regarded as outgrowths of the solution of the largest increasing subsequence problem for a random permutation. Also there are papers on financial correlations [132], random projections relating to phase retrieval [133], and on energy landscape statistics [134].

Already since the work of Dyson random matrix theories have been interpreted as one-dimensional integrable systems. In particular, the CalogeroSutherland model could be solved by means of techniques with their origin in random matrix theory. In this section we have one paper on the BC-type Calogero model [135]. More recently it was found that some random matrix theory partition functions are $\tau-$ functions of integrable hierarchies. This is explored in $[136,137,138]$. Other relations with integrable structures are discussed in [139].

Different types of integration formulae relevant to random matrix theory are discussed in section four. A very important class of integrals are the HarishChanda-Itzykson-Zuber integrals which are reviewed by Zinn-Justin and Zuber [140]. Other unitary matrix integrals are discussed in [141]. New results for ratios of determinants are obtained in [142].

The fifth section is on mesoscopic physics and disordered systems. Although this field is strongly rooted in experimental condensed matter physics we did not receive any such contributions. However, this section contains contains two theoretical reviews, one on transport through a chaotic quantum dot by Polianski and Brouwer [143] and one on wave function statistics by Merlin, Evers and Mildenberger [144]. Three different disordered systems are discussed, a two-sublattice system [145], a one-dimensional disordered system [146] and a microwave cavity [147].

The sixth section is on Non-Hermitian Random Matrix Theories. We can distinguish two different cases: weak non-hermiticity, which is reviewed by Fyodorov and Sommers [148], and strong non-hermiticity. In the first case the imaginary part of the eigenvalues is of the order of the spacing of the real parts. In the second case the real and imaginary parts of the eigenvalues are of the same order of magnitude which allows for much richer eigenvalue spectra than for Hermitian matrices (see [149], [150]). In addition to the review by Fyodorov and Sommers, eigenvalue correlations of different types of non-Hermitian matrices are studied in [151], [152] and [153]. The level spacings of a class of non-Hermitian matrices with real eigenvalues is studied in [154], while [155] treats the statistical properties of the zeros of a random analytic function which has close analogies with complex eigenvalues.

During the past two decades, quantum chaos has been one of the main anchors of random matrix theory. This special issue also has a section on this topic. One important issue has been to show (mostly numerically) that properties of simple chaotic systems can be described by random matrix theory. In this section we have a discussion of the eigenvalue spectra of the cat map [156], of scattering matrices on quantum graphs [157] and of action correlations and 
random matrix theory [158]. Wave functions of chaotic quantum systems are discussed in [159]. We also included in this section a paper on the calculation of parametric correlations [160] and a paper on random waves [161].

The applications of random matrix theories are by far not exhausted by the above mentioned sections. In the last section we have included random matrix theories that are not included in the previous section. We have included a review on embedded random matrix ensembles by Benet and Weidenmueller [162], and papers on distance matrices [163], the Penner model [164], the Wishart ensemble [165] and on critical random matrix models [166].

\section{Acknowledgments}

This work was partially supported by the Australian Research Council (P.J.F.), the Royal Society Dorothy Hodgkin Research Fellowship scheme (N.S.), and the US DOE grant DE-FG-88ER40388 (J.J.M.V.).

\section{References}

[1] T. Guhr, A. Mueller-Groeling, H.A. Weidenmueller, Random-matrix theories in quantum physics: common concepts, Phys. Rep. 299 (1998) 189.

[2] J. Wishart, Generalized product moment distribution in samples, Biometrika 20A (1928) 32 .

[3] E.P. Wigner, On the statistical distribution of the widths and spacings of nuclear resonance levels, Proc. Cam. Phil. Soc. 47 (1951) 790.

[4] E.P. Wigner, Characteristic vectors of bordered matrices with infinite dimensions Ann. Math. 62 (1955) 548.

[5] E.P. Wigner, Statistical properties of real symmetric matrices with many dimensions Can. Math. Congr. Proc., University of Toronto Press, Toronto 1957, p. 174.

[6] L. Landau and Ya. Smorodinsky, Lectures on the theory of the atomic nucleus, State Technical-Theoretical Literature Press, Moscow 1955, p. 92.

[7] I.I. Gurevich and M.I.Pevsner, Repulsion of Nuclear Levels, Nucl. Phys. 2 (1957) 575 .

[8] C.E. Porter and N. Rosenzweig, Statistical properties of atomic and nuclear spectra, Suomalaisen Tiedeakatemian Toimituksia AVI, 44 (1960). 
[9] M. Gaudin, Sur la loi limite de l'espacement des valeurs propres d'une matrice aléatoire, Nucl. Phys. 25 (1961) 447.

[10] M.L. Mehta, On the statistical properties of level spacings in nuclear spectra, Nucl. Phys. 18 (1960) 395.

[11] M.L. Mehta, L'emploi del polynomes orthogonaux pour calculer certain determinants, Rapport S.P.H. (Saclay) 658 (1960).

[12] F.J. Dyson, Statistical theory of the energy levels of complex systems, I, J. Math. Phys. 3 (1962) 140.

[13] F.J. Dyson, Statistical theory of the energy levels of complex systems, II, J. Math. Phys. 3 (1962) 157.

[14] F.J. Dyson, Statistical theory of the energy levels of complex systems, III, J. Math. Phys. 3 (1962) 166.

[15] F.J. Dyson, A Brownian motion model for the eigenvalues of a random matrix, J. Math. Phys. 3 (1962) 1191.

[16] F.J. Dyson, The threefold way. Algebraic structure of symmetry groups and ensembles in quantum mechanics, J. Math. Phys. 3 (1962) 1200.

[17] R. Balian, Random matrices and information theory, Nuovo Cimento B 57 (1968) 183.

[18] B. Sutherland, Exact results for a quantum many-body system in one dimension, Phys. Rev. A4 (1971) 2019.

[19] F. Calogero, Solution of one-dimensional $N$-body problems with quadratic and/or inversely quadratic pair potentials, J. Math. Phys. 12 (1971) 419.

[20] P.J. Forrester, Log-gases and random matrices, http://www.ms . unimelb.edu.au/〜matjpf/matpjf .html.

[21] V.E. Korepin, N.M. Bogoliubov and A.G. Izergin, Quantum inverse scattering method and correlation functions, Cambridge University Press, Cambridge, 1993.

[22] M.L. Mehta, Random matrices and the statistical theory of energy levels, Academic Press, New York 1967.

[23] C.E. Porter, Statistical theory of spectra: fluctuations, Academic Press, New York, 1965.

[24] P.W. Anderson, Absence of diffusion in certain random lattices, Phys. Rev. $109(1958) 1492$. 
[25] L.P. Gorkov and G.M. Eliasberg, Minute metallic particles in an electromagnetic field, Sov. Phys. JETP 21 (1965) 940.

[26] L.K. Hua, Harmonic analysis of functions of many complex variables in classical domains, Science Press, Peking, 1958.

[27] Harish-Chandra, Differential operators on a semi-simple Lie algebra, Amer. J. Math. 79 (1957) 85.

[28] C. Itzykson and J.-B. Zuber, Planar approximation 2, J. Math. Phys. 21 (1980) 411.

[29] A. Selberg, Bemerkninger om et multipelt integral, Nord. Mat. Tid. 26 (1944) 71.

[30] M.L. Mehta, Random matrices, 2nd edition, Academic Press, New York 1991.

[31] A.T. James, Zonal polynomials of real positive definite symmetric matrices, Ann. Math. 74 (1961) 456.

[32] R.J. Muirhead, Aspects of multivariate statistical theory, Wiley, New York, 1982.

[33] V.L. Girko, Theory of random determinants, Kluwer Academic Publishers, 1990.

[34] D. Voiculescu, Limit laws for random matrices and free products, Invent. Math. 104 (1991) 201.

[35] K.B. Efetov, Supersymmetry and the theory of disordered metals, Adv. Phys. 32 (1983) 53.

[36] K.B. Efetov, Level spacing distribution in small metallic particles, J. Phys. G15 (1982) L909.

[37] K.B. Efetov, Supersymmetric method in the localization theory, Zh. Eksp. Teor. Fiz. 82 (1982) 872.

[38] E.P. Wigner, On a class of analytic functions from the quantum theory of collisions, Ann. Math. 53 (1951) 36.

[39] C.E. Porter and R.G. Thomas, Fluctuations of nuclear reaction widths, Phys. Rev. 104 (1956) 483.

[40] T. Ericson, Fluctuations of nuclear cross-sections in the continuum region, Phys. Rev. Lett. 5 (1960) 430. 
[41] D. Agassi, Mantzouranis and H.A. Weidenmueller, The statistical theory of nuclear reactions for strongly overlapping resonances as a theory of transport phenomena, Phys. Rep. C22 (1975) 145.

[42] A.A. Abrikosov, L.P. Gorkov and I.E. Dzyaloshinski, Methods of quantum field theory in statistical physics, Prentice-Hall, Engelwood Cliffs, 1963.

[43] J. Ginibre, Statistical ensembles of complex, quaternion and real matrices, J. Math. Phys. 6 (1965) 440.

[44] H.L. Montgomery, The pair correlation of the zeta function, Proc. Symp. Pure Math. 24 (1973) 181.

[45] D.A. Hejhal, On the triple correlation of zeros of the zeta function, Inter. Math. Res. Notices 7 (1994) 293.

[46] Z. Rudnick and P. Sarnak, Zeros of principle L-functions and random matrix theory, Duke Math. J. 81 (1996) 269.

[47] E.B. Bogomolny and J.P. Keating, Random matrix theory and the Riemann zeros I: three- and four-point correlations, Nonlinearity 8 (1995) 1115.

[48] E.B. Bogomolny and J.P. Keating, Random matrix theory and the Riemann zeros II: n-point correlations, Nonlinearity 9 (1996) 911.

[49] N.M. Katz and P. Sarnak, Random matrices, Frobenius eigenvalues and monodromy, AMS, Providence, Rhode Island, 1999.

[50] J.B. Conrey, D.W. Farmer, J.P. Keating, M.O. Rubinstein and N.C. Snaith, Integral moments of zeta- and L-functions, preprint [arXiv:math.nt/0206018].

[51] J.B. Conrey, D.W. Farmer, J.P. Keating, M.O. Rubinstein and N.C. Snaith, Autocorrelation of random matrix polynomials, to be published in Comm. Math. Phys. [arXiv:math-ph/0208007].

[52] J.B. Conrey, J.P. Keating, M.O. Rubinstein and N.C. Snaith, On the frequency of vanishing of quadratic twists of modular L-functions, in Number Theory for the Millennium: Proceedings of the Millennial Conference on Number Theory, Ed. B.C. Berndt et al., A K Peters, Ltd, Boston (2001) [arXiv:math.nt/0012043].

[53] C.P. Hughes, J.P. Keating and N. O'Connell, Random matrix theory and the derivative of the Riemann zeta function, Proc. R. Soc. Lond. A 456 (2000) 2611.

[54] J.P. Keating and N.C. Snaith, Random matrix theory and $\zeta(1 / 2+i t)$, Comm. Math. Phys. 214 (2000) 57. 
[55] J.P. Keating and N.C. Snaith, Random matrix theory and L-functions at $s=1 / 2$, Comm. Math. Phys 214 (2000) 91.

[56] S.F. Edwards and P.W. Anderson, Theory of spin glasses, J. Phys. F - Met. Phys. 5 (1975) 965.

[57] F.J. Wegner, Mobility edge problem - continuous symmetry and a conjecture, Z. Phys. B35 (1979) 209.

[58] K.B. Efetov, A.I. Larkin and D.E. Khemlnitsky, Interaction of diffusion modes in the theory of localization, Zh. Eksp. Teor. Fiz. 79 (1980) 1120.

[59] A.J. McKane and M. Stone, Localization as an alternative to Goldstone theorem, Ann. Phys. 131 (1981) 36.

[60] L. Schaefer and F.J. Wegner, Disordered system with $N$-orbitals per site Lagrange formulation, hyperbolic symmetry, and Goldstone modes, Z. Phys B38 (1980) 113.

[61] D.J. Thouless, Maximum metallic resistance in thin wires, Phys. Rev. Lett. 39 (1977) 1167.

[62] J.J.M. Verbaarschot, H.A. Weidenmueller and M.R. Zirnbauer, Grassmann integration in stochastic quantum physics: the case of compound-nucleus scattering, Phys. Rep. 129 (1985) 367.

[63] F.J. Wegner, Algebraic derivation of symmetry-relations for disordered electronic systems, Z. Phys. B49 (1983) 297.

[64] B.D. Simons and B.L. Altshuler, Universal velocity correlations in disordered and chaotic systems, Phys. Rev. Lett. 70 (1993) 4063.

[65] K.B. Efetov, Supersymmetry in disorder and chaos, Cambridge University Press, Cambridge 1997.

[66] P.A. Mello, P. Pereyra and T. Seligman, Information-theory and statistical nuclear reactions 1. General theory and applications to few-channel problems, Ann. Phys. 161 (1985) 254.

[67] T.A. Brody, J. Flores, J.B. French, P.A. Mello, A. Pandey and S.S.M. Wong, Random-matrix physics - spectrum and strength fluctuations, Rev. Mod. Phys. 53 (1981) 385.

[68] R.A. Webb, S. Washburn, C.P. Umbach and R.B. Laibwitz, Observation of $H / E$ Ahoronov-Bohm oscillations in normal-metal rings, Phys. Rev. Lett. 54 (1985) 2696.

[69] B.L. Altshuler, Fluctuations in the extrinsic conductivity of disordered conductors, Pis'ma Zh. Eksp. Teor. Fiz. 41 (1985) 530. 
[70] A.D. Stone, Magnetoresistance fluctuations in mesoscopic wires and rings, Phys. Rev. Lett. 54 (1985) 2692.

[71] P.A. Lee and A.D. Stone, Universal conductance fluctuations in metals, Phys. Rev. Lett. 55 (1985) 1622.

[72] C.W.J. Beenakker, Random-matrix theory of quantum transport, Rev. Mod. Phys. 69 (1997) 731.

[73] Y. Alhassid, The statistical theory of quantum dots, Rev. Mod. Phys. 72 (2000) 895.

[74] G. 't Hooft, A two-dimensional model for mesons, Nucl. Phys. B 75 (1974) 461.

[75] E. Brezin, C. Itzykson, G. Parisi and J. B. Zuber, Planar diagrams, Comm. Math. Phys. 59 (1978) 35.

[76] P. Deift P, T. Kriecherbauer, K.T.R. McLaughlin, S. Venakides and X. Zhou, Uniform asymptotics for polynomials orthogonal with respect to varying exponential weights and applications to universality questions in random matrix theory, Comm. Pure and Appl. Math. 52 (1999) 1335.

[77] N. Seiberg and E. Witten, Electric - magnetic duality, monopole condensation, and confinement in N=2 supersymmetric Yang-Mills theory, Nucl. Phys. B 426 (1994) 19 [Erratum-ibid. B 430 (1994) 485] [arXiv:hepth/9407087].

[78] T. Eguchi and H. Kawai, Reduction of dynamical degrees of freedom in the large $N$ gauge theory, Phys. Rev. Lett. 48 (1982) 1063.

[79] S. Weinberg, Pion scattering lengths, Phys. Rev. Lett. 17 (1966) 616.

[80] J. Gasser and H. Leutwyler, Light quarks at low temperatures, Phys. Lett. B184 (1987) 83.

[81] E. V. Shuryak and J. J. Verbaarschot, Random matrix theory and spectral sum rules for the Dirac operator in QCD, Nucl. Phys. A 560 (1993) 306 [arXiv: hep-th/9212088].

[82] J. J. Verbaarschot, The spectrum of the QCD Dirac operator and chiral random matrix theory: The threefold way, Phys. Rev. Lett. 72 (1994) 2531 [arXiv:hep-th/9401059].

[83] R. Gade, Anderson localization for sublattice models, Nucl. Phys. B 398 (1993) 398.

[84] P.J. Forrester, The spectrum edge of random matrix ensembles, Nucl. Phys. B402 (1993) 709. 
[85] J. J. Verbaarschot and T. Wettig, Random matrix theory and chiral symmetry in QCD, Ann. Rev. Nucl. Part. Sci. 50 (2000) 343 [arXiv:hep$\mathrm{ph} / 0003017]$.

[86] R. Oppermann, Anderson localization problems in gapless superconducting phases, Physica A167 (1990) 301.

[87] A. Altland, M.R. Zirnbauer, Random matrix theory of a chaotic Andreev quantum dot, Phys. Rev. Lett. 76 (1996) 3420.

[88] F.J. Dyson, Correlations between the eigenvalues of a random matrix, Comm. Math. Phys. 19 (1970) 235.

[89] M.R. Zirnbauer, Riemannian symmetric superspaces and their origin in random-matrix theory, J Math. Phys. 37 (1996) 4986.

[90] V. A. Kazakov and A. A. Migdal, Induced QCD at large N, Nucl. Phys. B 397 (1993) 214 [arXiv:hep-th/9206015].

[91] R. Dijkgraaf and C. Vafa, Matrix models, topological strings, and supersymmetric gauge theories, Nucl. Phys. B 644 (2002) 3 [arXiv:hep-th/0206255].

[92] F. David, Planar diagrams, two-dimensional lattice gravity and surface models, Nucl. Phys. B 257 (1985) 45.

[93] V. A. Kazakov, A. A. Migdal and I. K. Kostov, Critical properties of randomly triangulated planar random surfaces, Phys. Lett. B 157 (1985) 295.

[94] M. R. Douglas and S. H. Shenker, Strings in less than one-dimension, Nucl. Phys. B 335 (1990) 635.

[95] E. Brezin and V. A. Kazakov, Exactly solvable field theories of closed strings, Phys. Lett. B 236 (1990) 144.

[96] D. J. Gross and A. A. Migdal, Nonperturbative two-dimensional quantum gravity, Phys. Rev. Lett. 64 (1990) 127.

[97] J. Ambjorn, J. Jurkiewicz and Y. M. Makeenko, Multiloop correlators for two-dimensional quantum gravity, Phys. Lett. B 251 (1990) 517.

[98] M. J. Bowick and E. Brezin, Universal scaling of the tail of the density of eigenvalues in random matrix models, Phys. Lett. B 268 (1991) 21.

[99] G. Akemann, P. H. Damgaard, U. Magnea and S. Nishigaki, Universality of random matrices in the microscopic limit and the Dirac operator spectrum, Nucl. Phys. B 487 (1997) 721 [arXiv:hep-th/9609174].

[100] M. R. Douglas, Strings in less than one-dimension and the generalized $K-D$-V hierarchies, Phys. Lett. B 238 (1990) 176. 
[101] R. Dijkgraaf, H. Verlinde and E. Verlinde, Loop equations and Virasoro constraints in nonperturbative 2-d quantum gravity, Nucl. Phys. B 348 (1991) 435.

[102] P. Di Francesco, P. Ginsparg and J. Zinn-Justin, 2-D Gravity and random matrices, Phys. Rep. 254 (1995) 1 [arXiv:hep-th/9306153].

[103] M. Jimbo, T. Miwa, Y. Môri and M. Sato, Density matrix of an impenetrable Bose gas and the fifth Painlevé transcendent, Physica 1D (1980) 80 .

[104] C.A. Tracy and H. Widom, Level spacing distributions and the Airy kernel, Comm. Math. Phys. 159 (1994) 151.

[105] J. Baik, P. Deift and K. Johannsson, On the distribution of the length of the longest increasing subsequence of random permutations, J. Amer. Math. Soc. 36 (1999) 1119.

[106] M. Prähofer and H. Spohn, Universal distributions for growth processes in one dimension and random matrices, Phys. Rev. Lett. 84 (2000) 4882.

[107] K. Johansson. Non-intersecting paths, random tilings and random matrices, Prob. Theory Rel. Fields 123 (2002) 225.

[108] G.M. Zaslavsky, Stochasticity in quantum systems, Phys. Rep. 80 (1981) 157.

[109] M.V. Berry, Quantizing a classically ergodic system: Sinai's billiard and the KKR method, Ann. Phys. 131 (1981) 163.

[110] O. Bohigas, M.J. Giannoni and C. Schmit, Characterization of chaotic quantum spectra and universality of level fluctuation laws, Phys. Rev. Lett. $52(1984) 1$.

[111] Ya.G. Sinai, Dynamical systems with elastic reflections. Ergodic properties of dispersing billiards, Russ. Math. Surv. 25 (1970) 137.

[112] E. Haller, H, Koppel and L.S. Cederbaum, Uncovering the transition from regularity to irregularity in a quantum system, Phys. Rev. Lett. 52 (1984) 1665.

[113] T. Seligman, J.J.M. Verbaarschot and M.R. Zirnbauer, Quantum chaos and the transition from chaotic to regular motion, Phys. Rev. Lett. 53 (1984) 215.

[114] M.V. Berry, Semiclassical theory of spectral rigidity, Proc. Roy. Soc. A 400 (1985) 229. 
[115] E.B. Bogomolny and J.P. Keating, Gutzwiller's trace formula and spectral statistics, Phys. Rev. Lett. 77 (1996) 1472.

[116] F. Haake, Quantum signatures of chaos, 2nd edition, Springer, Berlin 1999.

[117] H.-J. Stoeckmann, Quantum chaos: an introduction, Cambridge University Press, Cambridge 1999.

[118] L. Laloux, P. Cizeau, J.-P. Bouchard and M. Potters, Noise dressing of financial correlation matrices, Phys. Rev. Lett. 83 (1999) 1467.

[119] V. Plerou, P. Gopikrishnan, B. Rosnow L.A. Nunes Amaral and H.E. Stanley, Universal and nonuniversal properties of cross correlations in financial time series, Phys. Rev. Lett. 83 (1999) 1471.

[120] A.L. Moustakas, H.U. Baranger, L. Balents A.M. Sengupta and S.H. Simon, Communication through a diffusive medium: coherence and capacity, Science, 287 (2000) 287 [arXiv:cond-mat/0009097].

[121] M. Coram and P. Diaconis, New tests of the correspondence between unitary eigenvalues and the zeros of Riemann's zeta function.

[122] C.P. Hughes and Z. Rudnick, Mock-Gaussian behaviour for linear statistics of classical compact groups, [arXiv:math.pr/0206289].

[123] C.P. Hughes and Z. Rudnick, Linear statistics of low-lying zeros of Lfunctions, [arXiv:math.nt/0208230].

[124] C.P. Hughes and Z. Rudnick, Linear statistics for zeros of Riemann's zeta Function, [arXiv:math.nt/0208220].

[125] C.P. Hughes, Random matrix theory and discrete moments of the Riemann zeta function, [arXiv:math.nt/0207236].

[126] J.P. Keating, N. Linden and Z. Rudnik, Random matrix theory, the exceptional Lie groups and L-functions, [arXiv:math-ph/0210058].

[127] F. Mezzadri, Random matrix theory and the zeros of $\zeta^{\prime}(s)$, [arXiv:math$\mathrm{ph} / 0207044]$.

[128] J.P. Keating and N. Snaith, Random matrices and L-functions.

[129] K. Hikami and T. Imamura, Vicious walkers and hook Young tableaux, [arXiv:cond-mat/0209512].

[130] N. O'Connell, Conditioned random walks and the RSK correspondence.

[131] A. Borodin and P.J. Forrester, Increasing subsequences and the hard-tosoft edge transition in matrix ensembles [arXiv:math-ph/0205007]. 
[132] T. Guhr and B. Kaelber, A new method to estimate the noise in financial correlation matrices, [arXiv:cond-mat/0206577].

[133] V. Elser, Random projections and the optimization of an algorithm for phase retrieval.

[134] M. Degli Esposti, C. Giardinà and S. Graffi, Energy landscape statistics of the random orthogonal model [arXiv:cond-mat/0207681].

[135] S.M. Nishigaki, D.M. Gangardt and A. Kamenev, Correlation functions of the BC Calogero-Sutherland model [arXiv:cond-mat/0207301].

[136] M. Bertola, B. Eynard and J. Harnad, Partition functions for matrix models and isomonodromic tau functions, [arXiv:nlin.SI/0204054].

[137] V.A. Kazakov and A. Marshakov, Complex curve of the two matrix model and its tau-function [arXiv:hep-th/0211236].

[138] I.K. Kostov, Integrable flows in c=1 string theory, [arXiv:hep-th/0208034].

[139] P. Bleher and B. Eynard Double scaling limit in random matrix models and a nonlinear hierarchy of differential equations, [arXiv:hep-th/0209087].

[140] P. Zinn-Justin and J.-B. Zuber, On some integrals over the $U(N)$ unitary group and their large $N$ limit [arXiv:math-ph/0209019].

[141] B. Schlittgen and T. Wettig, Generalizations of some integrals over the unitary group, [arXiv:math-ph/0209030].

[142] Y.V. Fyodorov and E. Strahov, An exact formula for general spectral correlation function of random Hermitian matrices, [arXiv:math-ph/0204051].

[143] M.L. Polianski and P.W. Brouwer, Scattering matrix ensemble for time-dependent transport through a chaotic quantum dot, [arXiv:condmat/0208408].

[144] A.D. Mirlin, F. Evers and A. Mildenberger, Wave function statistics and multifractality at the spin quantum Hall transition, [arXiv:condmat/0208451].

[145] S.N. Evangelou and D.E. Katsanos, Spectral statistics in chiral-orthogonal disordered systems, [arXiv:cond-mat/0206089].

[146] J. Richert and H.A. Weidenmüller, Localization properties of two interacting electrons in a disordered quasi one-dimensional potential, [arXiv:condmat/0206520].

[147] R. Schaefer, T. Gorin, T.H. Seligman and H.-J. Stoeckmann, Correlation functions of scattering matrix elements in microwave cavities with strong absorption [arXiv:nlin.CD/0212021]. 
[148] Y.V. Fyodorov and H.-J Sommers Random matrices close to Hermitian or unitary: overview of methods and results, [arXiv:nlin.CD/0207051].

[149] D.E. Holz, H. Orland and A. Zee, On the remarkable spectrum of a nonHermitian random matrix model, [arXiv:math-ph/0204015].

[150] K. Zyczkowski, M. Kus, W. Slomczyriski and H.-J. Sommers, Random unistochastic matrices.

[151] G. Akemann, The solution of a chiral random matrix model with complex eigenvalues, [arXiv:hep-th/0204246].

[152] P. Wiegmann and A. Zabrodin, Large scale correlations in normal and general non-Hermitian matrix ensembles, [arXiv:hep-th/0210159].

[153] B. Rider, A limit theorem at the edge of a non-Hermitian random matrix ensemble.

[154] Z. Ahmed and S.R. Jain, Gaussian ensemble of $2 \times 2$ pseudo-Hermitian random matrices.

[155] M.R. Dennis and J.H. Hannay, Saddle points in the chaotic analytic function and Ginibre characteristic polynomial, [arXiv:nlin.CD/0209056]

[156] A. Gamburd, J. Lafferty and D. Rockmore, Eigenvalue spacings for quantized cat maps.

[157] T. Kottos and U. Smilansky, Quantum graphs: A simple model for chaotic scattering, [arXiv:nlin.CD/0207049].

[158] U. Smilansky and B. Verdene, Action correlations and random matrix theory. [arXiv:nlin.CD/0208034].

[159] N.R. Cerruti and S. Tomsovic, A uniform approximation for the fidelity in chaotic systems, [arXiv:nlin.CD/0210030].

[160] I.E. Smolyarenko and B.D. Simons, Parametric statistics of individual energy levels in random Hamiltonians [arXiv:cond-mat/0207456].

[161] D. Hejhal and H. Christianson, On correlations of CM-type Maas waveforms under the horocyclic flow.

[162] L. Benet and H.A. Weidenmüller, Review of the $k$-body embedded ensembles of Gaussian random matrices, [arXiv:cond-mat/0207656].

[163] E. Bogomolny, O. Bohigas and C. Schmit, Spectral properties of distance matrices. [arXiv:nlin.CD/0301044].

[164] N. Deo, Large N Penner matrix model and a novel asymptotic ansatz for the generalized Laguerre polynomials. 
[165] R.A. Janik and M.A. Nowak, Wishart and anti-Wishart random matrices, [arXiv:math-ph/0112017].

[166] M.L. Ndawana and V.E. Kravtsov, Energy level statistics of a critical random matrix ensemble. [arXiv:cond-mat/0302569]. 